\title{
REVIEW
}

\section{Transplantation of Cryopreserved Teeth: A Systematic Review}

\author{
Thanaphum Osathanon* \\ Department of Anatomy, Faculty of Dentistry, Chulalongkorn University, Bangkok, Thailand
}

\begin{abstract}
The aim of this article was to examine the research articles regarding biological and mechanical properties of cryopreserved teeth for potential use in tooth transplantation. A systematic review of literatures was performed by Pubmed searching with assigned key words from January 1, 1990 to June 8, 2009. All articles were examined for inclusion criteria. Secondary search was conducted by hand-search through references of included articles from primary search.

A total of 24 articles were obtained from both primary and secondary search and used as fundamental articles in this review. Periodontal ligament tissues of cryopreserved teeth were able to maintain their biological properties resulted in a satisfactory healing of periodontium. Dental pulp tissues,
\end{abstract}

however, may be compromised by limitation of permeability of cryopreservative agent into pulp cavity. Therefore, an endodontic treatment of transplanted cryopreserved teeth was recommended. Cryopreserved teeth had comparable mechanical properties to those of normal teeth. Importantly, the success of cryopreserved tooth transplantation treatment in orthodontic patients was reported.

The cryopreserved teeth for tooth banking have a potential clinical application for treatment of missing teeth. Case selection, however, is critical for treatment success. More studies and data regarding masticatory function and periodontal healing of transplanted cryopreserved teeth are needed.

Keywords cryopreservation, transplantation, teeth

\section{Introduction}

Recently, methods have been reported in a number of studies for growing biological teeth in the laboratory to replace missing teeth (Ohazama et al., 2004; Yen and Sharpe 2006; Ferreira et al., 2007; Duailibi et al., 2008; Abukawa et al., 2009; Zhang et al., 2009). Cells isolated from tooth buds seeded on biodegradable scaffolds are able to form tooth-like structures containing dentin-pulp complex, enamel and cementum (Young et al., 2002). The sequential seeding of epithelial and mesenchymal cells has been used to improve the ability to control morphology of bioengineered teeth. Upon transplantation of the bioengineered tooth in extracted socket, reinnervation and revas- cularization have been observed in the dental pulp of bioengineered teeth (Nakao et al., 2007). The eruption and function of bioengineering molar tooth germ in oral cavity was recently reported (Ikeda et al., 2009). These data strongly suggest the potential for tooth replacement using biological engineered teeth. The success observed in these studies, however, is still limited by the lack of embryonic cell source and the difficulty in engineering complex tooth morphology (e.g., molar teeth).

In addition to conventional prosthetic and dental implants, autotransplantation is an alternative treatment for treating missing teeth. Case selection plays a crucial role, however, in the success of transplantation. A seventy-nine percent success 
rate was reported in patients underwent autotransplantation treatment in long term follow up (17-41 years) (Czochrowska et al., 2002). The success of tooth autotransplantation has been reported in iliac grafts for mandible reconstruction after odotogenic myxoma resection (Landes et al., 2008), and in conjunction with guided bone tissue regeneration (Hurzeler and Quinones 1993). Transplantation of immature teeth results in higher success rates. The success of tooth transplantation relies on several factors (i.e., the initial stability, the extraoral time, the surgical procedure, as well as the handling and care of periodontal ligament (Kim et al., 2005)). Common complications of tooth transplantation are observed, such as root resorption and ankylosis (Kim et al., 2005). Therefore, it has been suggested that root canal treatment should be performed in transplanted teeth with complete root formation to prevent root resorption (Claus et al., 2004; Azevedo et al., 2007).

The idea of cryopreserved teeth for further autotransplantation when needed has been introduced as alternative choice to conventional prosthetic, dental implants, and even bioengineered teeth due to the technical, economical and functional issues (Oh et al., 2005). Teeth to be extracted for orthodontic reasons, impacted third molars, or embedded teeth are excellent candidates to cryopreserve for future transplantation. In this review, the biological and mechanical properties of cryopreserved teeth are evaluated. The potential uses of these cryopreserved teeth in clinical management of missing teeth are also discussed.

\section{Materials and Methods}

The Pubmed database was searched in the period of January 1, 1990 - June 8, 2009 using keywords ("1990/01/01" [Publication Date]: "2009/ 06/08" [Publication Date]) AND ((cryopreserved or cryopreservation) and (teeth or tooth)). Publications were limited to English. The author examined the abstracts of the articles for inclusion and exclusion criteria. References of included articles were further examined to identify additional publications that followed the inclusion criteria. Criteria for inclusion were as follows: 1) articles published in English peer-reviewed journals, 2) in vitro, in vivo and clinical studies, which examined methods for tooth cryopreservation, biology of cryopreserved tooth and mechanical aspects of cryopreserved tooth. The exclusion was any study evaluating cryopreservation of tooth for specimen storage/preservation for dental material or mechanical tests.

\section{Results}

Using the described search strategy, 108 articles were identified from Pubmed by the search criteria. A total of ten articles were entered for evaluation. From examination of the references of the included articles, and additional 15 articles were added. A total of 25 articles were used in this review.

\section{Discussion and Literature Review}

\section{Freezing methods for the cryopreservation of the teeth}

Ice injury is a major concern for tissue cryopreservation. Kawasaki et al. suggested that the slow and rate-controlling freezing reduced the ice injury of cryopreserved living cells (Kawasaki et al., 2004). Cryoprofiles including type, concentration, equilibration and dilution of cryoprotective agent as well as freezing rate and thawing conditions were need to be optimized for specific cell or tissues (Schwartz 1992). The transplantation of slow freezing teeth resulted in periodontal regeneration similar to immediate transplantation whereas the rapid freezing teeth resulted in progressive root resorption (Kawasaki et al., 2004). Moreover, Bartlett et al. reported that cryopreservation of developing teeth, using slow rate cooling, resulted in normal dental hard and soft tissue formation. Also, this cryopreservation protocol did not compromise survival and development of tooth germ (Bartlett and Reade, 1972).

Dimethylsulfoxide (DMSO) has been commonly used in many studies as the ingredient for the freezing medium. DMSO prevents cytoplasmic membrane destruction from intracellular ice crystal formation during the freezing process. The adverse 
effect of this reagent, however, has also been published. Ten percent DMSO has been reported as the optimal concentration in freezing medium and shown to have better effects in protecting the periodontal ligament during cryopreservation (Schwartz et al., 1985) resulting in minimal ankylosis (Schwartz and Andreasen, 1983). Schwartz et al. suggested the optimal cryoprofile for mature teeth that should be the stepwise equilibration at room temperature to final concentration in ten percent DMSO and then a slow freezing rate at $0.3{ }^{\circ} \mathrm{C} \cdot \mathrm{min}^{-1}$ to $-35^{\circ} \mathrm{C}$, followed by plunging in liquid nitrogen (Schwartz et al., 1985). The detailed laboratory and clinical procedure required for cryopreservation of teeth has been previously described by Schwartz (Schwartz 1992).

\section{Biological responses of cryopreserved teeth periodontal ligament}

Viability and functionality of periodontal ligament are crucial parameters for the success of tooth transplantation. Upon subcutaneous implantation, short-term cryopreserved teeth exhibited similar results to those of immediate transplanted teeth (Kawasaki et al., 2004; Izumi et al., 2007). At the first week, granulation tissue formation around the cryopreserved teeth was noted in associated with infiltration of inflammatory cells (Kawasaki et al., 2004; Izumi et al., 2007). Cementum predominated with acellular cementum (Izumi et al., 2007). A decrease in cementoblast number was also observed (Izumi et al., 2007). The remaining periodontal ligament remaining on the root surface was positively stained for alkaline phosphatase, suggesting the viability and potential differentiation function of periodontal ligament cells (Kawasaki et al., 2004). At the second week after transplantation, the regeneration of periodontium was noted (Izumi et al., 2007). Cementoblasts and fibroblasts were increases in number at root surface (Kawasaki et al., 2004). The alveolar bone formation was noted around the root with the formation of periodontal ligament (Izumi et al., 2007). No ankylosis was found (Kawasaki et al., 2004; Izumi et al., 2007). Several immunopositive cathepsin K cells (Izumi et al., 2007) or Tartate resistant acid phosphatase (TRAP) positive cells (Kawasaki et al., 2004) were found on the root surface of transplanted cryopreserved teeth, implying formation of osteoclasts and potential resorption and remodeling of periodontium. No obvious resorption, however, was observed.

Together, these data suggest the excellent periodontal healing of transplanted cryopreserved tooth. The transplanted cryopreserved tooth had, however, a delayed periodontal tissue regeneration compared to those of immediate transplanted teeth. This observation suggests that the cryopreservation procedure may somewhat compromise the viability and functionality of periodontal cells. Izumi et al. stated that the cryopreserved teeth in a deep freezer at $-152^{\circ} \mathrm{C}$ might decrease the damage to periodontal tissues more so than those in $-80^{\circ} \mathrm{C}$ (Izumi et al., 2007). The enlargement of epithelial rests of Malassez in the cryopreserved teeth after transplantation in rat abdomen was shown to be the result of inflammation and cryoprotectant (Kawasaki et al., 2004). Seo et al. showed the normal histological structure of periodontal ligament after the cryopreservation in liquid nitrogen for three and six months (Seo et al., 2005). No positive terminal deoxynucleotidyl transferase dUTP nick end labeling (TUNEL) staining was observed (Seo et al., 2005).

The periodontal ligament stem cell can be isolated from cryopreserved periodontal ligament (Seo et al., 2005). These cells maintained their characteristics, i.e., expression of surface marker, single colony strain generation, osteogenic/cementogenic and adipocytes differentiation, and cementun/periodontal ligament-like tissue regeneration upon subcutaneous implantation in immunocompromised mice (Seo et al., 2005). The number of single colonies derived from cryopreserved periodontal ligament, however, was significantly decreased (Seo et al., 2005). Temmerman et al. showed that membrane integrity of periodontal ligament (PDL) cells, growth capacity, and alkaline phosphatase (ALP) activity were similar between PDL fibroblast isolated from cryopreserved teeth and fresh extracted teeth (Temmerman et al., 2006). Detailed information on periodontal healing after immediate transplanted teeth compared to cryopreserved transplanted teeth has been extensively reviewed by Temmerman et al. (Temmerman et al., 2006). 


\section{Dental pulp}

It has been reported that the dental pulp might undergo necrosis after transplantation of cryopreserved mature teeth due to the difficulty in diffusion of cryprotectant medium into pulp tissue (Schwartz and Andreasen, 1983). Therefore, root canal treatment was considered to be the necessary procedure. In one case of transplantation of an immature tooth, revascularization in pulp chamber was reported (Laureys et al., 2001). In dogs, the transplanted cryopreserved immature teeth and the mature apicoectomized teeth had no significant differences in revascularization compared to immediate transplantation using microangiography evaluation, suggesting that cryopreservation did not impair the revascularization process (Laureys et al., 2001). The quality of the new vascular tissue formation was comparable to control as the subdentinal capillary plexus could be observed (Laureys et al., 2001). In addition, Kawasaki et al. reported that the revascularized areas in the pulp were positive for alkaline phosphatase, suggesting potential functioning of dental pulp cells (Kawasaki et al., 2004). It was also reported that the success rate of revascularization was higher in incisors compared to premolars (Laureys et al., 2001). Interestingly, Laureys et al. also reported that revascularization can be observed in pulp chamber of transplanted teeth whose pulp tissue was removed completely off after tooth extraction (Laureys et al., 2001). Therefore, these researchers suggested this technique of revascularization for transplantation of cryopreserved teeth, as it might prevent inflammatory resorption and eliminate the root canal treatment procedure after transplantation (Laureys et al., 2001).

Various publications have reported the success of isolation of dental pulp stem cells from cryopreserved teeth. The viable dental pulp stem cells were obtained from cryopreserved teeth in liquid nitrogen for 1 month (Perry et al., 2008). Similarities in growth, surface marker expression and differentiation were observed between dental pulp stem cells obtained from cryopreserved and fresh extracted teeth (Perry et al., 2008). These data showed that the cryopreservation procedure did not compromise viability of dental pulp tissue, even though the diffusion of cryopreservation medium into dental pulp was limited.

\section{Physical properties of cryopreserved teeth}

When cryopreservation was performed at $-196^{\circ} \mathrm{C}$ for 1 week, no crack or microfracture was observed in the cryopreserved teeth (Oh et al., 2005). The hardness test exhibited similar results of dental tissue after thawing the cryopreserved teeth compared to control in both before and after placement in a wet chamber at $37^{\circ} \mathrm{C}$ for 1 week (Oh et al., 2005). Twenty-five percent of cryopreserved teeth, however, exhibited a longitudinal fracture during hardness testing (Oh et al., 2005). Panighi et al. reported that the shear bond strength of mid-coronal dentin was not compromised by cryopreservation procedure (Panighi et al., 1997). The significant difference of the shear dentin bond strength was observed, however, in the superficial dentin (Panighi et al., 1997). Together, these results illustrate comparable mechanical properties between cryopreserved and normal teeth. Clinical function (e.g., chewing ability) is crucial in terms of mechanical performance of teeth, but no evidence of mechanical function of cryopreserved teeth in the clinical situation has been reported.

\section{Clinical application of cryopreserved teeth}

The indication of cryopreserved teeth is when immediate positioning of grafts is not possible, but the future transplantation site can be determined (Schwartz, 1992). The transplantation of cryopreserved teeth would be the suitable choice for treatment of missing teeth in children and adolescence, since it has been shown that the transplanted teeth retain the potential induction of alveolar bone growth during the eruption process (Day et al., 2008). The transmission of marginal gingiva with gingival papillae has also been possible (Paulsen et al., 2006). Moreover, the superior properties of transplanted teeth to those dental implants were reported. First, functional periodontal ligament of transplanted teeth is restored, unlike in dental implants, and this regeneration of periodontal ligament is crucial for various aspects, such as orthodontic movement and rotation for adjusting position of teeth (Paulsen et al., 2006; Day et al., 2008), induction of alveolar bone remodeling and 


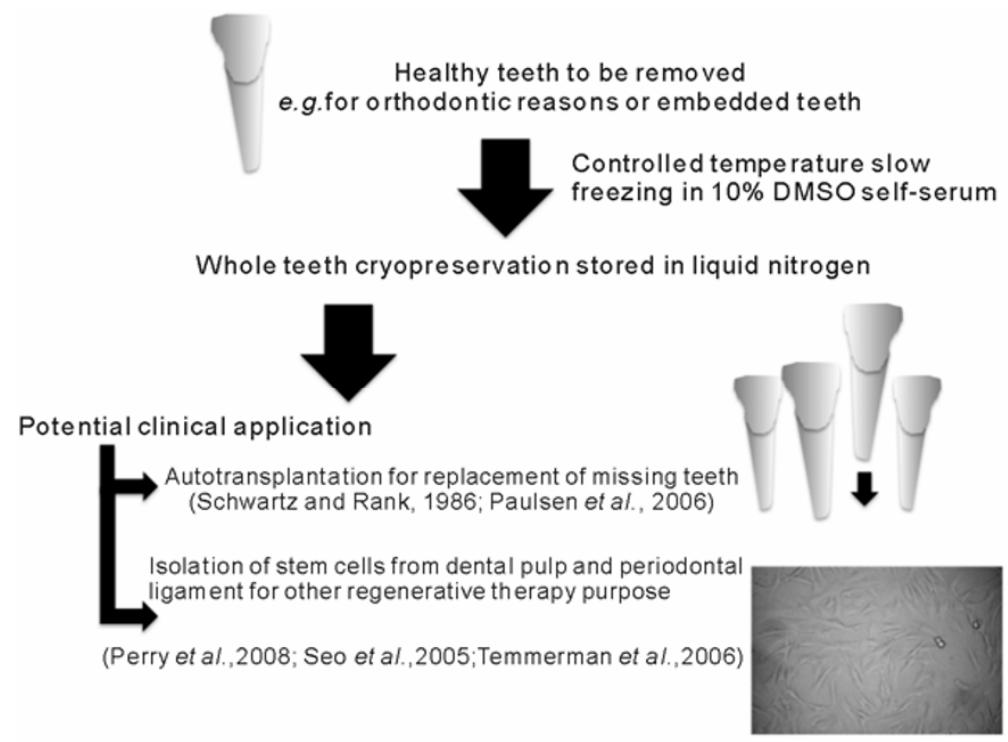

Figure 1 The strategy of potential application of cryopreserved tooth

growth (Paulsen et al., 2006), and preventing excessive chewing damage due to nociceptive nerve ending. Second, transplanted teeth do not need the unnecessary preparation of the sound tooth structure, which was the major disadvantage of the conventional prosthetic treatment. Third, transplanted teeth have a potential to continue eruption. With respect to dental implants, the marginal bone loss around the adjacent teeth and buccal to the implants, as well as the infraoccluded implanted-supported crown, may be observed due to the continuous eruption of the adjacent teeth and growth of craniofacial structure, especially in adolescence patients (Thilander et al., 2001). The strategy of potential application of cryopreserved tooth is illustrated in Figure 1.

Schwartz et al. reported the success of autotransplanted cryopreserved first left premolar in the space obtained from orthodontic treatment. Four years after transplantation, the transplanted tooth appeared clinically and radiographically healthy, with no sign of root resorption (Schwartz and Rank, 1986; Schwartz, 1992). Schwartz also reported the similar PDL healing of cryopreserved tooth compared to those of unfrozen teeth (Schwartz, 1992). Recently, Paulsen et al. combined the cryopreservation of premolar as an reserve donor tooth in the plan for treating complicated trauma in maxillary region of eight years old patient (Paulsen et al., 2006). Autotransplantation of cryopreserved tooth has been reported as a treatment procedure for several cases, including replacement of traumatized tooth and missing tooth in the cleft area (Schwartz, 1992).

The potential application for transplantation of cryopreserved teeth is promising. Autotransplantation of cryopreserved teeth might be the perfect application. The idea of tooth banking for allografts has also been introduced. Yang et al. reported the use of premolars from a tooth bank for replacement of central incisors (Yang and Chang, 1990). Adequate apical healing and minimal replacement resorption was observed after 3 years (Yang and Chang, 1990). The authors did not discuss immunological reactions to transplanted cryopreserved tooth allografts. But as with other allografts, the risk of blood borne transmitted disease and immunological reaction are a concern. Anneroth et al. described the inflammation and root resorption of allotransplantation of teeth in monkeys, suggesting the possible allograft rejection of transplanted teeth (Anneroth et al., 1988). Moreover, the study of subcutaneous implantation of teeth allotransplantation in rabbits illustrated that allotransplantation evoked the inflammatory response. The authors suggested that periodontal ligament could be a major source for the antigenic stimulation involved in graft rejection (Birman and de Araujo, 1975). Despite the disadvantages and biological issues of tooth allotransplantation, Smetanka and Cooper raised the ethics and potential risks of allotransplantation are similar to those of xenotransplan- 
tation (e.g., the illegal trade and the risk of infection transfer (Smetanka and Cooper, 2005)). Together, these data might suggest that the most suitable application for cryopreserved tooth is for the autotransplantation application.

For clinical application of cryopreserved teeth, the medium used for storing teeth during cooling procedure is very important. The serum is added in those media to help increase the survival rate of cells by providing growth factors as well as nutrients. Though, using bovine or fetal calf serum, an immunological reaction to bovine protein remaining on the transplanted teeth might occur. The autologous serum is suggested for mixing in the cryopreservation medium, as this is recommended in those procedures for cell-based bone tissue engineering. In addition, the control freezing temperature of the specimens is also critical. The standard freezing machine should be used to control decrease temperature at a constant rate before storing the sample in the liquid nitrogen. Failure of the freezing procedure may result in impaired cell survival which leads to an unpredictable clinical outcome.

\section{Conclusion}

Cryopreserved teeth for tooth banking have a potential clinical application for the treatment of missing teeth. Periodontal ligament healing of cryopreserved teeth is shown to be biological satisfactory even though the root canal treatment is still suggested for better success of transplanted teeth. Mechanical properties of cryopreserved teeth are slightly compromise but still comparable to those of normal teeth. Importantly, case selection is critical for treatment success. More evidence regarding the masticatory function and periodontal healing of transplanted cryopreserved tooth in clinical situation requires further investigation.

\section{Acknowledgements}

The author was supported by Young Investigator Research Promotion Grant, Chulalongkorn University.

\section{References}

Abukawa H, Zhang W, Young CS, Asrican R, Vacanti JP, Kaban LB, et al. (2009). Reconstructing mandibular defects using autologous tissue-engineered tooth and bone constructs. J Oral Maxillofac Surg, 67(2): 335347.

Anneroth G, Lundquist G, Nordenram A, SÖder PO (1988). Re- and allotransplantation of teeth - an experimental study in monkeys. Int J Oral Maxillofac Surg, 17(1): 54-57.

Azevedo PC, Moura CC, Zanetta-Barbosa D, Bernadineli $\mathrm{N}$ (2007). Time of endodontic treatment in autogenic transplants of mature teeth: histological study in dogs. Oral Surg Oral Med Oral Pathol Oral Radiol Endod, 104(2): 287-293.

Bartlett PF, Reade PC (1972). Cryopreservation of developing teeth. Cryobiology, 9(3): 205-211.

Birman EG, de Araujo NS (1975). Autotransplants and allotransplants of teeth in the subcutaneous tissue of rabbits: a histological study. J Dent Res, 54(3): 508514.

Claus I, Laureys W, Cornelissen R, Dermaut LR (2004). Histologic analysis of pulpal revascularization of autotransplanted immature teeth after removal of the original pulp tissue. Am J Orthod Dentofacial Orthop, 125(1): 93-99.

Czochrowska EM, Stenvik A, Bjercke,B, Zachrisson BU (2002). Outcome of tooth transplantation: survival and success rates 17-41 years posttreatment. Am J Orthod Dentofacial Orthop, 121(2): 110-119.

Day PF, Kindelan SA, Spencer JR, Kindelan JD, Duggal MS (2008). Dental trauma: part 2. Managing poor prognosis anterior teeth - treatment options for the subsequent space in a growing patient. J Orthod, 35(3): 143-155.

Duailibi SE, Duailibi MT, Zhang W, Asrican R, Vacanti JP, Yelick PC (2008). Bioengineered dental tissues grown in the rat jaw. J Dent Res, 87(8): 745-750.

Ferreira CF, Magini RS, Sharpe PT (2007). Biological tooth replacement and repair. J Oral Rehabil, 34(12): 933-939.

Hurzeler MB, Quinones CR (1993). Autotransplantation of a tooth using guided tissue regeneration. J Clin Periodontol, 20(7): 545-548.

Ikeda E, Morita R, Nakao K, Ishida K, Nakamura T, Takano-Yamamoto T, et al. (2009). Fully functional bioengineered tooth replacement as an organ replacement therapy. Proc Natl Acad Sci U S A, 106(32): 
13475-13480.

Izumi N, Yoshizawa M, Ono Y, Kobayashi T, Hamamoto Y, Saito C (2007). Periodontal regeneration of transplanted rat teeth subcutaneously after cryopreservation. Int $J$ Oral Maxillofac Surg, 36(9): 838-844.

Kawasaki N, Hamamoto Y, Nakajima T, Irie K, Ozawa H (2004). Periodontal regeneration of transplanted rat molars after cryopreservation. Arch Oral Biol, 49(1): 59-69.

Kim E, Jung JY, Cha IH, Kum KY, Lee SJ (2005). Evaluation of the prognosis and causes of failure in 182 cases of autogenous tooth transplantation. Oral Surg Oral Med Oral Pathol Oral Radiol Endod, 100(1): 112-119.

Landes CA, Glasl B, Ludwig B, Rieger J, Sader R (2008). Tooth autotransplantation in a free iliac crest graft for prosthetic reconstruction. J Craniofac Surg, 19(5): 1281-1286.

Laureys W, Beele H, Cornelissen R, Dermaut L (2001). Revascularization after cryopreservation and autotransplantation of immature and mature apicoectomized teeth. Am J Orthod Dentofacial Orthop, 119(4): 346-352.

Nakao K, Morita R, Saji Y, Ishida K, Tomita Y, Ogawa M, et al. (2007). The development of a bioengineered organ germ method. Nat Methods, 4(3): 227-230.

Oh YH, Che ZM, Hong JC, Lee EJ, Lee SJ, Kim J (2005). Cryopreservation of human teeth for future organization of a tooth bank - a preliminary study. Cryobiology, 51(3): 322-329.

Ohazama A, Modino SA, Miletich I, Sharpe PT (2004). Stem-cell-based tissue engineering of murine teeth. $J$ Dent Res, 83(7): 518-522.

Panighi MM, Allart D, Jacquot BM, Camps J, G'Sell C (1997). Influence of human tooth cryopreservation on dentin bond strength. Dent Mater, 13(1): 56-61.

Paulsen HU, Andreasen JO, Schwartz O (2006). Tooth loss treatment in the anterior region: autotransplantation of premolars and cryopreservation. World J Orthod, 7(1): 27-34.

Perry BC, Zhou D, Wu X, Yang FC, Byers MA, Chu TM, et al. (2008). Collection, cryopreservation, and characterization of human dental pulp-derived mesenchymal stem cells for banking and clinical use. Tissue Eng Part C Methods, 14(2): 149-156.
Schwartz O, Andreasen JO (1983). Cryopreservation of mature teeth before replantation in monkeys. I. Effect of different cryoprotective agents and freezing devices. Int J Oral Surg, 12(6): 425-436.

Schwartz O, Andreasen JO, Greve T (1985). Cryopreservation before replantation of mature teeth in monkeys. II. Effect of preincubation, different freezing and equilibration rates and endodontic treatment upon periodontal healing. Int J Oral Surg, 14(4): 350-361.

Schwartz O, Rank CP (1986). Autotransplantation of cryopreserved tooth in connection with orthodontic treatment. Am J Orthod Dentofacial Orthop, 90(1): 67-72.

Schwartz O (1992). Cryopreservation of teeth before replantation or transplantation // Andreasen JO ed. Atlas of replantation and transplantation of teeth. Freibourg: Mediglobe SA, p 241-256.

Seo BM, Miura M, Sonoyama W, Coppe C, Stanyon R, Shi S (2005). Recovery of stem cells from cryopreserved periodontal ligament. J Dent Res, 84(10): 907-912.

Smetanka C, Cooper DK (2005). The ethics debate in relation to xenotransplantation. Rev Sci Tech, 24(1): $355-342$.

Temmerman L, De Pauw G.A, Beele H, Dermaut LR (2006). Tooth transplantation and cryopreservation: state of the art. Am J Orthod Dentofacial Orthop, 129(5): 691-695.

Thilander B, Odman J, Lekholm U (2001). Orthodontic aspects of the use of oral implants in adolescents: a 10-year follow-up study. Eur J Orthod, 23(6): 715-731.

Yang ZP, Chang CS (1990). A 3-year follow-up of a homotransplanted tooth from a tooth bank. J Endod, 16(1): 34-37.

Yen AH, Sharpe PT (2006). Regeneration of teeth using stem cell-based tissue engineering. Expert Opin Biol Ther, 6(1): 9-16.

Young CS, Terada S, Vacanti JP, Honda M, Bartlett JD, Yelick PC (2002). Tissue engineering of complex tooth structures on biodegradable polymer scaffolds. J Dent Res, 81(10): 695-700.

Zhang W, Abukawa H, Troulis MJ, Kaban LB, Vacanti JP, Yelick PC (2009). Tissue engineered hybrid tooth-bone constructs. Methods, 47(2): 122-128.

*Corresponding author: Thanaphum Osathanon

Address: Department of Anatomy, Faculty of Dentistry, Chulalongkorn University, Henri-Dunant Rd., Pathumwan, Bangkok 10330, Thailand

Tel: $022188885 \quad$ Fax: $022188870 \quad$ E-mail: osathanon.t@gmail.com 